\title{
Global Optimization for the Sum of Certain Nonlinear Functions
}

\author{
Mio Horai, ${ }^{1}$ Hideo Kobayashi, ${ }^{1}$ and Takashi G. Nitta $^{2}$ \\ ${ }^{1}$ Faculty of Engineering, Graduate School of Engineering, Mie University, Kurimamachiyamachi, Tsu 514-8507, Japan \\ ${ }^{2}$ Department of Education, Mie University, Kurimamachiyamachi, Tsu 514-8507, Japan
}

Correspondence should be addressed to Mio Horai; mio@com.elec.mie-u.ac.jp

Received 13 April 2014; Revised 19 August 2014; Accepted 20 August 2014; Published 10 November 2014

Academic Editor: Julio D. Rossi

Copyright (c) 2014 Mio Horai et al. This is an open access article distributed under the Creative Commons Attribution License, which permits unrestricted use, distribution, and reproduction in any medium, provided the original work is properly cited.

We extend work by Pei-Ping and Gui-Xia, 2007, to a global optimization problem for more general functions. Pei-Ping and Gui-Xia treat the optimization problem for the linear sum of polynomial fractional functions, using a branch and bound approach. We prove that this extension makes possible to solve the following nonconvex optimization problems which Pei-Ping and Gui-Xia, 2007, cannot solve, that the sum of the positive (or negative) first and second derivatives function with the variable defined by sum of polynomial fractional function by using branch and bound algorithm.

\section{Introduction}

The optimization problem is widely used in sciences, especially in engineering and economy [1-3]. In 2007, Pei-Ping and Gui-Xia considered one global optimization problem in [4]:

$$
\begin{array}{ll}
\min & \omega(x)=\sum_{j=1}^{P} c_{j} \frac{b_{j}(x)}{a_{j}(x)} \\
\text { s.t. } & g_{k}(x) \leq 0, \quad x \in X,
\end{array}
$$

where $X:=\left\{x \in \mathbf{R}^{N} \mid 0<\underline{x}_{i} \leq x_{i} \leq \bar{x}_{i}<\infty(i=\right.$ $1,2, \ldots, N)\}, a_{j}(x), b_{j}(x), g_{k}(x)$ are given generalized polynomial. One has

$$
\begin{gathered}
a_{j}(x):=\sum_{t=1}^{T_{j}^{a}} \beta_{j t}^{a} \prod_{i=1}^{N} x_{i}^{\gamma_{j t i}^{a}}, \quad b_{j}(x):=\sum_{t=1}^{T_{j}^{b}} \beta_{j t}^{b} \prod_{i=1}^{N} x_{i}^{\gamma_{j t i}^{b}}, \\
g_{k}(x):=\sum_{t=1}^{T_{k}^{g}} \beta_{k t}^{a} \prod_{i=1}^{N} x_{i}^{\gamma_{k t i}^{g}} .
\end{gathered}
$$

Sum of rations problems like $(P)$ attract a lot of attention, and the reason is that these problems are applied to various economical problems [4].
Pei-Ping and Gui-Xia proposed the method to solve these problems globally by using branch and bound algorithm in [4]. In the above problem, the objective function and constrained function are sums of generalized polynomial fractional functions. We extend these functions to more general functions like below:

$$
\begin{array}{ll}
\min & w(x)=\sum_{j=1}^{P} h_{j}\left(\frac{b_{j}(x)}{a_{j}(x)}\right) \\
\text { s.t. } & g_{k}(x)=\sum_{j=1}^{P_{k}} h_{k j}\left(\frac{d_{k \dot{j}}(x)}{c_{k j}(x)}\right) \leq 0 \\
& \left(\dot{j}=1, \ldots, P_{K}, k=1, \ldots, M\right), \\
& x \in X,
\end{array}
$$

where $a_{j}(x)>0, b_{j}(x)>0, c_{k j}(x)>0, d_{k j}(x)>0 x \in X$; that is,

$$
\begin{gathered}
a_{j}(x):=\sum_{t=1}^{T_{j}^{a}} \beta_{j t}^{a} \prod_{i=1}^{N} x_{i}^{\gamma_{j t i}^{a}}, \quad b_{j}(x):=\sum_{t=1}^{T_{j}^{b}} \beta_{j t}^{b} \prod_{i=1}^{N} x_{i}^{\gamma_{j t i}^{b}}, \\
c_{k j}(x):=\sum_{t=1}^{T_{k j}^{c}} \beta_{k j t}^{c} \prod_{i=1}^{N} x_{i}^{\gamma_{k j t}^{c}},
\end{gathered}
$$




$$
\begin{gathered}
d_{k \dot{j}}(x):=\sum_{t=1}^{T_{k j}^{d}} \beta_{k j t}^{d} \prod_{i=1}^{N} x_{i}^{\gamma_{k j t}^{d}}, \\
\left(j=1,2, \ldots, P, \dot{j}=1,2, \ldots, P_{k}, k=1,2, \ldots, M\right),
\end{gathered}
$$

where $T_{j}^{a}, T_{j}^{b}, T_{k j}^{c}, T_{k j}^{d}$ are natural numbers, and $\beta_{j t}^{a}, \beta_{j t}^{b}, \beta_{k j t}^{c}$, $\beta_{k j t}^{d}$ are real constants not zero, and $\gamma_{j t i}^{a}, \gamma_{j t i}^{b}, \gamma_{k j t}^{c}, \gamma_{k j t}^{d}$ are real constants.

We assume that $h_{j}\left(y_{j}\right), h_{k j}\left(y_{k \dot{j}}\right): \mathbf{R} \mapsto \mathbf{R}$ are secondly differentiable functions and monotone increasing or monotone decreasing functions. We divide these functions to monotone increasing or monotone decreasing as follows:

$$
\begin{gathered}
h_{j}^{\prime}>0 \quad(j=1, \ldots, K), \quad h_{j}^{\prime}<0 \quad(j=K+1, \ldots, P), \\
h_{k j}^{\prime}>0 \quad\left(\dot{j}=1, \ldots, K_{k}\right), \\
h_{k j}^{\prime}<0 \quad\left(\dot{j}=K_{k}+1, \ldots, P_{k}\right) .
\end{gathered}
$$

Furthermore, we assume the following conditions for the second derivatives:

$$
\begin{gathered}
\left\{h_{j} \circ \exp \left(z_{j}\right)\right\}^{\prime \prime}>0, \quad\left\{h_{j} \circ \exp \left(z_{j}\right)\right\}^{\prime \prime}<0, \\
\left\{h_{k^{\prime}} \circ \exp \left(z_{k j}\right)\right\}^{\prime \prime}>0, \quad\left\{h_{k j^{\circ}} \circ \exp \left(z_{k \dot{j}}\right)\right\}^{\prime \prime}<0 \\
\left(j=1, \ldots, P, \quad j=1, \ldots, P_{k}, k=1, \ldots, M\right) .
\end{gathered}
$$

To solve the above problem $(P 0)$, we transform the problem $(P 0)$ to the equivalent problems $(P 1),(P 2)$ and transform $(P 2)$ into the linear relaxation problem. We prove the equivalency of the problems under above assumption, and we calculate the equivalent problem using branch and bound algorithm corresponding to [4-6].

For example, according to this extension at approach, we can calculate the following global optimization problem:

$$
\begin{array}{ll}
\min & \sin \left(\frac{x_{1}^{2}+3 x_{2}-2 x_{2}^{2}+1}{x_{1}^{2}+x_{2}+2}\right) \\
& +\cos \left(\frac{-x_{2}^{2}+2 x_{1}+2 x_{2}}{x_{1}+2.5}\right) \\
\text { s.t. } & x_{1}^{2}-\frac{x_{1}}{x_{2}}-1 \leq 0 \\
& x_{1}+3 \frac{x_{2}}{x_{1}}-5 \leq 0 \\
& X=\left\{x: 1 \leq x_{1} \leq 3,1 \leq x_{2} \leq 3\right\} .
\end{array}
$$

In this paper, we explain how to make equivalent relaxation linear problem from original problem in Section 2. In Section 3, we present the branch and bound algorithm and its convergence. In Section 4, we introduce numerical experiments result.

\section{Equivalence Transformation and Linear Relaxation}

In this section we firstly transform the problem $(P 0)$ to the equivalent problems $(P 1)$ and secondly transform $(P 1)$ to $(P 2)$. Thirdly we linearize the problem $(P 2)$ corresponding to $[4]$.

2.1. Translation of the Problem (P0) into $(P 1)$. For the problem $(P 0)$, we put new variables $m_{j}, l_{j}, t_{k j}$ and $s_{k \dot{j}}$, and the function $\rho(l, m)$ and $\xi_{k}(s, t)$ depending on $h_{j}, h_{k j}$ in the original problem $(P 0)$ :

$$
\begin{gathered}
\rho(l, m):=\sum_{j=1}^{P} h_{j}\left(\frac{m_{j}}{l_{j}}\right) \quad(j=1,2, \ldots, P), \\
\xi_{k}(s, t):=\sum_{j=1}^{P_{k}} h_{k j}\left(\frac{t_{k j}}{s_{k j}}\right) \quad\left(j=1, \ldots, P_{k}, k=1, \ldots, M\right) .
\end{gathered}
$$

Since $a_{j}(x), b_{j}(x), c_{k j}(x), d_{k^{\prime}}(x)$ are polynomials on closed interval $X$, it is easy to calculate the minimums and maximums of the functions on $X$; we denote them by $\underline{a}_{j}, \bar{a}_{j}, \underline{b}_{j}, \bar{b}_{j}$, $\underline{c}_{k j}, \bar{c}_{k j^{\prime}}, \underline{d}_{k j^{\prime}}, \bar{d}_{k j}$.

Let $H$ be the closed interval:

$$
\begin{aligned}
H:=\{ & (l, m, s, t) \in \mathbf{R}^{P_{\text {sum }}} \\
& \underline{a}_{j} \leq l_{j} \leq \bar{a}_{j}, \underline{b}_{j} \leq m_{j} \leq \bar{b}_{j}, \\
& \left.\underline{c}_{k j} \leq s_{k j} \leq \bar{c}_{k j^{\prime}}, \underline{d}_{k j} \leq t_{k j} \leq \bar{d}_{k \dot{j}}\right\},
\end{aligned}
$$

where $P_{\text {sum }}=2\left(P+\sum_{k=1}^{M} P_{k}\right)$.

Let $Z_{H}$ be the following closed domain in $X \times H$; that is,

$$
\begin{gathered}
Z_{H}:=\left\{\begin{array}{l}
(x, l, m, s, t) \in X \times H \mid \\
\xi_{k}(s, t) \leq 0 \quad(k=1, \ldots, M), \\
l_{j}-a_{j}(x) \leq 0, \quad b_{j}(x)-m_{j} \leq 0 \\
\quad(j=1, \ldots, K), \\
a_{j}(x)-l_{j} \leq 0, \quad m_{j}-b_{j}(x) \leq 0
\end{array}\right. \\
s_{k j}-c_{k j}(x) \leq 0, \quad d_{k j}(x)-t_{k j} \leq 0 \\
\quad\left(j=1, \ldots, K_{k}, k=1, \ldots, M\right), \\
c_{k j}(x)-s_{k j} \leq 0, \quad t_{k j}-d_{k j}(x) \leq 0 \\
\left.\left(j=K_{k}+1, \ldots, P_{k}, k=1, \ldots, M\right)\right\} .
\end{gathered}
$$


We give the problem $(P 1)$ on $Z_{H}$. Consider

$$
\begin{array}{ll}
\min & \rho(l, m) \\
\text { s.t. } & \xi_{k}(s, t) \leq 0, \quad(k=1, \ldots, M), \\
& (x, l, m, s, t) \in Z_{H} .
\end{array}
$$

Now we obtain Theorem 1 that proves the equivalence of $(P 0)$ and $(P 1)$.

Theorem 1. The problem (P0) on $X$ is equivalent to the problem $(P 1)$ on $Z_{H}$.

Proof. Let $x^{*}$ be the optimal solution for $(P 0)$; we denote

$$
\begin{aligned}
l_{j}^{*}:=a_{j}\left(x^{*}\right), & m_{j}^{*}:=b_{j}\left(x^{*}\right), \\
s_{k j}^{*}:=c_{k j}\left(x^{*}\right), & t_{k^{\prime}}^{*}:=d_{k^{\prime} j}\left(x^{*}\right),
\end{aligned}
$$

and then

$$
\begin{aligned}
& \sum_{j=1}^{P} h_{j}\left(\frac{b_{j}\left(x^{*}\right)}{a_{j}\left(x^{*}\right)}\right)=\sum_{j=1}^{P} h_{j}\left(\frac{m_{j}^{*}}{l_{j}^{*}}\right), \\
& \sum_{j=1}^{P_{k}} h_{k j}\left(\frac{d_{k^{\prime}}\left(x^{*}\right)}{c_{k j}\left(x^{*}\right)}\right)=\sum_{j=1}^{P_{k}} h_{k j}\left(\frac{t_{k j}^{*}}{s_{k j}^{*}}\right) .
\end{aligned}
$$

Furthermore let $\left(x^{\sharp}, l^{\sharp}, m^{\sharp}, s^{\sharp}, t^{\sharp}\right)$ be the optimal solution for $(P 1)$. Then by the restricted condition we have the following:

for $j=1, \ldots, K, 0<l_{j}^{\sharp} \leq a_{j}\left(x^{\sharp}\right)$ and $0<b_{j}\left(x^{\sharp}\right) \leq m_{j}^{\sharp}$; that is, $0<b_{j}\left(x^{\sharp}\right) / a_{j}\left(x^{\sharp}\right) \leq m_{j}^{\sharp} / l_{j}^{\sharp}$;

for $j=K+1, \ldots, P, 0<a_{j}\left(x^{\sharp}\right) \leq l_{j}^{\sharp}$ and $0<m_{j}^{\sharp} \leq$ $b_{j}\left(x^{\sharp}\right)$; that is, $0<m_{j}^{\sharp} / l_{j}^{\sharp} \leq b_{j}\left(x^{\sharp}\right) / a_{j}\left(x^{\sharp}\right)$;

for $\dot{j}^{\prime}=1, \ldots, K_{k}$ and $k=1, \ldots, M, 0<s_{k j}^{\sharp} \leq c_{k j}\left(x^{\sharp}\right)$ and $0<d_{k j}\left(x^{\sharp}\right) \leq t_{k j}^{\sharp}$; that is, $0<d_{k j}\left(x^{\sharp}\right) / c_{k j}\left(x^{\sharp}\right) \leq$ $t_{k \dot{j}}^{\#} / s_{k j}^{\#} ;$

for ${ }^{\prime}=K_{k}+1, \ldots, P_{k}$ and $k=1, \ldots, M, 0<c_{k j}\left(x^{\sharp}\right) \leq$ $s_{k j}^{\#}$ and $0<t_{k j}^{\#} \leq d_{k j}\left(x^{\sharp}\right)$; that is, $0<t_{k j}^{\sharp} / s_{k j}^{\#} \leq$ $d_{k j}\left(x^{\sharp}\right) / c_{k j}\left(x^{\sharp}\right)$.

The conditions $h_{j}^{\prime}>0(j=1, \ldots, K)$, or $h_{j}^{\prime}<0(j=K+$ $1, \ldots, P)$ and $h_{k j}^{\prime}>0\left(j=1, \ldots, K_{k}\right)$, or $h_{k j}^{\prime}<0\left(j^{\prime}=K_{k}+\right.$ $\left.1, \ldots, P_{k}\right)$ lead to

$$
\begin{gathered}
h_{j}\left(\frac{b_{j}\left(x^{\sharp}\right)}{a_{j}\left(x^{\sharp}\right)}\right) \leq h_{j}\left(\frac{m_{j}^{\sharp}}{l_{j}^{\sharp}}\right) \quad(j=1, \ldots, P), \\
h_{k j}\left(\frac{d_{k j}\left(x^{\sharp}\right)}{c_{k j}\left(x^{\sharp}\right)}\right) \leq h_{k j}\left(\frac{t_{k j}^{\sharp}}{s_{k j}^{\sharp}}\right) \\
\left(j=1, \ldots, P_{k}, k=1, \ldots, M\right) .
\end{gathered}
$$

Therefore we obtain

$$
\begin{gathered}
\sum_{j=1}^{P} h_{j}\left(\frac{b_{j}\left(x^{\sharp}\right)}{a_{j}\left(x^{\sharp}\right)}\right) \leq \sum_{j=1}^{P} h_{j}\left(\frac{m_{j}^{\#}}{l_{j}^{\sharp}}\right), \\
\sum_{j=1}^{P_{k}} h_{k j}\left(\frac{d_{k j}\left(x^{\sharp}\right)}{c_{k j}\left(x^{\sharp}\right)}\right) \leq \sum_{j=1}^{P_{k}} h_{k j}\left(\frac{t_{k j}^{\#}}{s_{k j}^{\sharp}}\right) .
\end{gathered}
$$

Now,

$$
\sum_{j=1}^{P_{k}} h_{k \dot{j}}\left(\frac{d_{k \dot{j}}\left(x^{\sharp}\right)}{c_{k j}\left(x^{\sharp}\right)}\right) \leq \sum_{j=1}^{P_{k}} h_{k \dot{j}}\left(\frac{t_{k \dot{j}}^{\#}}{s_{k j}^{\sharp}}\right) \leq 0,
$$

that is, $x^{\sharp}$ satisfied constant for $(P 0)$.

Since the optimal solution for $(P 0)$ is $x^{*}$, we obtain

$$
h_{j}\left(\frac{b_{j}\left(x^{*}\right)}{a_{j}\left(x^{*}\right)}\right) \leq h_{j}\left(\frac{b_{j}\left(x^{\sharp}\right)}{a_{j}\left(x^{\sharp}\right)}\right),
$$

so

$$
\begin{gathered}
\sum_{j=1}^{P} h_{j}\left(\frac{m_{j}^{*}}{l_{j}^{*}}\right)=\sum_{j=1}^{P} h_{j}\left(\frac{b_{j}\left(x^{*}\right)}{a_{j}\left(x^{*}\right)}\right) \leq \sum_{j=1}^{P} h_{j}\left(\frac{m_{j}^{\sharp}}{l_{j}^{\sharp}}\right), \\
\sum_{j=1}^{P} h_{j}\left(\frac{m_{j}^{*}}{l_{j}^{*}}\right) \leq \sum_{j=1}^{P} h_{j}\left(\frac{m_{j}^{\sharp}}{l_{j}^{\sharp}}\right) .
\end{gathered}
$$

For the optimal solution $x^{*}$ of $(P 0)$, we denote

$$
\begin{aligned}
l_{j}^{*}:=a_{j}\left(x^{*}\right), & m_{j}^{*}:=b_{j}\left(x^{*}\right), \\
s_{k j}^{*}:=c_{k j}\left(x^{*}\right), & t_{k^{\prime}}^{*}:=d_{k j}\left(x^{*}\right) ;
\end{aligned}
$$

then

$$
\rho\left(l^{*}, m^{*}\right)=w\left(x^{*}\right), \quad \xi_{k}\left(s^{*}, t^{*}\right)=g\left(x^{*}\right) .
$$

The element $\left(x^{*}, l^{*}, m^{*}, s^{*}, t^{*}\right)$ satisfies the conditions for $Z_{H}$. Since $\left(x^{\sharp}, l^{\sharp}, m^{\sharp}, s^{\sharp}, t^{\sharp}\right)$ is the optimal solution for $(P 1)$, it satisfies $\sum_{j=1}^{P} h_{j}\left(m_{j}^{\sharp} / l_{j}^{\sharp}\right) \leq \sum_{j=1}^{P} h_{j}\left(m_{j}^{*} / l_{j}^{*}\right)$.

Hence $\sum_{j=1}^{P} h_{j}\left(m_{j}^{\sharp} / l_{j}^{\sharp}\right)=\sum_{j=1}^{P} h_{j}\left(m_{j}^{*} / l_{j}^{*}\right)$; that is, the two problems are equivalent.

2.2. Translation of the Problem (P1) into $(P 2)$. We change the variables by the logarithmic function $\log$. Since $x_{i}, l_{j}, m_{j}, s_{k j}$, $t_{k j^{\prime}}$ are positive, we can write $x_{i}, l_{j}, m_{j}, s_{k j^{\prime}}, t_{k^{\prime} j^{\prime}}$ as $\exp \left(y_{n}\right)$ are using new variables $y_{n}\left(n=1, \ldots, N+P_{\text {sum }}\right)$; that is, $y_{i}:=$ $\ln x_{i}, y_{N+j}:=\ln l_{j}, y_{N+P+j}:=\ln m_{j}, y_{N+2 P+(k-1) P_{k}+j}:=\ln s_{k j}$, and $y_{N+2 P+(M+k-1) P_{k}+j}:=\ln t_{k j}$. 
The closed domain $Z_{H}$ corresponds to the following $S^{0}$, where

$$
\begin{aligned}
S^{0}:=\{ & y \in \mathbf{R}^{N+P_{\text {sum }}} \\
& \ln \underline{x}_{i} \leq y_{i} \leq \ln \bar{x}_{i}, \\
& \ln \underline{a}_{j} \leq y_{N+j} \leq \ln \bar{a}_{j}, \\
& \ln \underline{b}_{j} \leq y_{N+P+j} \leq \ln \bar{b}_{j}, \\
& \ln \underline{c}_{k^{\prime}} \leq y_{N+2 P+(k-1) P_{k}+j} \leq \ln \bar{c}_{k^{\prime}}, \\
& \left.\ln \underline{d}_{k j} \leq y_{N+2 P+(M+k-1) P_{k j}} \leq \ln \bar{d}_{k{ }^{\prime}}\right\} .
\end{aligned}
$$

Using such transformation of variables, the objective function and the restricted functions of $(P 1)$ are changed to the following:

$$
\begin{aligned}
& \sum_{j=1}^{P} h_{j}\left(\frac{m_{j}}{l_{j}}\right)=\sum_{j=1}^{P} h_{j} \circ \exp \left(y_{N+P+j}-y_{N+j}\right), \\
& \sum_{j=1}^{P_{k}} h_{k j}\left(\frac{t_{k \dot{j}}}{s_{k \dot{j}}}\right) \\
& =\sum_{j=1}^{P_{k}} h_{k \dot{j}} \circ \exp \left(y_{N+2 P+(M+k-1) P_{k}+\dot{j}}-y_{N+2 P+(k-1) P_{k}-\dot{j}}\right), \\
& l_{j}-a_{j}(x)=\exp \left(y_{N+j}\right)-\sum_{t=1}^{T_{j}^{a}} \beta_{j t}^{a} \exp \left(\sum_{i=1}^{N} \gamma_{j t i}^{a} y_{i}\right), \\
& b_{j}(x)-m_{j}=\sum_{t=1}^{T_{j}^{b}} \beta_{j t}^{b} \exp \left(\sum_{i=1}^{N} \gamma_{j t i}^{b} y_{i}\right)-\exp \left(y_{N+P+j}\right), \\
& s_{k \dot{j}}-c_{k \dot{j}}(x)=\exp \left(y_{N+2 P+(k-1) P_{k}+\dot{j}}\right) \\
& -\sum_{t=1}^{T_{j}^{c}} \beta_{j t}^{c} \exp \left(\sum_{i=1}^{N} \gamma_{j t i}^{c} y_{i}\right) \\
& d_{k j^{\prime}}(x)-t_{k \dot{j}}=\sum_{t=1}^{T_{j}^{d}} \beta_{j t}^{d} \exp \left(\sum_{i=1}^{N} \gamma_{j t i}^{d} y_{i}\right) \\
& -\exp \left(y_{N+2 P+(M+k-1) P_{K}+\dot{j}}\right) \text {. }
\end{aligned}
$$

Now $\rho(l, m), \xi_{k}(s, t), l_{j}-a_{j}(x), b_{j}(x)-m_{j}, s_{k j}-c_{k j}(x)$, $d_{k \dot{j}}(x)-t_{k \dot{j}}$, are represented as

$$
\sum_{t=1}^{T_{m}} \Psi_{m t} \circ \exp \left(\sum_{i=1}^{N+P_{\text {sum }}} \lambda_{m t i} y_{i}\right)
$$

where $\lambda_{m t i}$ is real number and $\Psi_{m t}$ satisfies $\Psi_{m t}^{\prime}(x)>0$ or $\Psi_{m t}^{\prime}(x)<0$, and $\left\{\Psi_{m t} \circ \exp (y)\right\}^{\prime \prime}>0$ or $\left\{\Psi_{m t} \circ \exp (y)\right\}^{\prime \prime}<0$.

Let $f_{m t}(y)$ be $\Psi_{m t} \circ \exp (y)$ and let $\mu_{m}(y)$ be $\sum_{t=1}^{T_{m}} f_{m t}\left(\sum_{i=1}^{N+P_{\text {sum }}} \lambda_{m t i} y_{i}\right)$.
Then the objective function $\rho(l, m)$ and the restricted functions are changed functions which are changed to $\mu_{0}(y)$ and $\mu_{m}(y)\left(m=1, \ldots, M+2\left(P+\sum_{k=1}^{M} P_{k}\right)\right)$.

Now we put

$S_{\mu}^{0}:=\left\{y \in S^{0} \mid\right.$

$$
\left.\mu_{m}(y) \leq 0\left(m=1,2, \ldots, M+2\left(P+\sum_{k=1}^{M} P_{k}\right)\right)\right\}
$$

Then the problem $(P 1)$ is transformed naturally to the following problem $(P 2)$ :

$$
\begin{array}{ll}
\min & \mu_{0}(y) \\
\text { s.t. } & y \in S_{\mu}^{0} .
\end{array}
$$

2.3. Linearization of the Problem (P2). The objective and restricted function for $(P 2)$ are nonlinear. On $S_{\mu}^{0}$, we approximate $\mu_{m}(y)$ to lower bounded linear functions, and we can transform $(P 2)$ into the linear optimization problem. The solution of it is lower bound of the optimal value on $(P 2)$. We denote $\underline{y}_{i}, \bar{y}_{i}, \underline{y}_{N+j}, \bar{y}_{N+j}, \underline{y}_{N+P+j}, \bar{y}_{N+P+j}, \underline{y}_{N+2 P+(k-1) P_{k}+\hat{j}}$, $\bar{y}_{N+2 P+(k-1) P_{k}+\hat{j}}, \underline{y}_{N+2 P+(M+k-1) P_{k}+\hat{j}}, \bar{y}_{N+2 P+(M+k-1) P_{k}+\hat{j}}$ as minimums and maximums for $\ln \underline{x}_{i}, \ln \bar{x}_{i}, \ln \underline{a}_{j}, \ln \bar{a}_{j}, \ln \underline{b}_{j}, \ln \bar{b}_{j}$, $\ln \underline{c}_{k j^{\prime}}, \ln \bar{c}_{k j^{\prime}}, \ln \underline{d}_{k j^{\prime}}, \ln \bar{d}_{k j}\left(j=1, \ldots, P,{ }^{\prime}=1, \ldots, P_{k}, k=\right.$ $1, \ldots, M)$.

And we denote $S^{q} \subset S_{\mu}^{0}$; that is,

$$
\begin{aligned}
& S^{q}:=\left\{y \in \mathbf{R}^{N+P_{\text {sum }}} \mid\right. \\
& \underline{y}_{i} \leq \underline{y}_{i}^{q} \leq y_{i} \leq \bar{y}_{i}^{q} \leq \bar{y}_{i} \\
& \left.\left(i=1, \ldots, N+P_{\text {sum }}\right)\right\}, \\
& Y_{m t}^{S^{q}}:=\sum_{i=1}^{N+P_{\text {sum }}} \lambda_{m t i} y_{i} \\
& \underline{Y}_{m t}^{S^{q}}:=\sum_{i=1}^{N+P_{\text {sum }}} \min \left\{\lambda_{m t i} \underline{y}_{i}^{q}, \lambda_{m t i} \bar{y}_{i}^{q}\right\}, \\
& \bar{Y}_{m t}^{S^{q}}:=\sum_{i=1}^{N+P_{\text {sum }}} \max \left\{\lambda_{m t i} \underline{y}_{i}^{q}, \lambda_{m t i} \bar{y}_{i}^{q}\right\}, \\
& \left(m=0,1,2, \ldots, M+2\left(P+\sum_{k=1}^{M} P_{k}\right), t=1, \ldots, T_{m}\right) .
\end{aligned}
$$

Now, $f_{m t}^{\prime}(y)>0$ or $f_{m t}^{\prime}(y)<0$, and $f_{m t}^{\prime \prime}(y)>0$ or $f_{m t}^{\prime \prime}(y)<0$, and $f_{m t}\left(Y_{m t}^{S^{q}}\right)$ is monotonic convex function on $\left[\underline{Y}_{m t}^{S^{q}}, \bar{Y}_{m t}^{S^{q}}\right]$. And there exist the upper and lower bounded linear functions $\left(F_{m t}^{S^{q}}\left(Y_{m t}^{S^{q}}\right)\right.$ and $\left.G_{m t}^{S^{q}}\left(Y_{m t}^{S^{q}}\right)\right)$ of $f_{m t}\left(Y_{m t}^{S^{q}}\right)$. 
We denote

$$
\begin{aligned}
F_{m t}^{S^{q}}\left(Y_{m t}^{S^{q}}\right):= & \frac{f_{m t}\left(\bar{Y}_{m t}^{S^{q}}\right)-f_{m t}\left(\underline{Y}_{m t}^{S^{q}}\right)}{\bar{Y}_{m t}^{S^{q}}-\underline{Y}_{m t}^{S^{q}}}\left(Y_{m t}^{S^{q}}-\underline{Y}_{m t}^{S^{q}}\right) \\
& +f_{m t}\left(\underline{Y}_{m t}^{S^{q}}\right) .
\end{aligned}
$$

As $f_{m t}\left(Y_{m t}^{S^{q}}\right)$ is continuous on $\left[\underline{Y}_{m t}^{S^{q}}, \bar{Y}_{m t}^{S^{q}}\right]$ and differentiable on $\left(\underline{Y}_{m t}^{S^{q}}, \bar{Y}_{m t}^{S^{q}}\right)$, there exists $c_{m t}^{S^{q}} \in\left(\underline{Y}_{m t}^{S^{q}}, \bar{Y}_{m t}^{S^{q}}\right)$, such that

$$
f_{m t}^{\prime}\left(c_{m t}^{S^{q}}\right)=\frac{f_{m t}\left(\bar{Y}_{m t}^{S^{q}}\right)-f_{m t}\left(\underline{Y}_{m t}^{S^{q}}\right)}{\bar{Y}_{m t}^{S^{q}}-\underline{Y}_{m t}^{S^{q}}},
$$

by the mean value theorem.

Since $f_{m t}^{\prime}(y)>0$ or $f_{m t}^{\prime}(y)<0, f_{m t}\left(Y_{m t}^{S^{q}}\right)$ is monotonic function on $\left[\underline{Y}_{m t}^{S^{q}}, \bar{Y}_{m t}^{S^{q}}\right]$, there exists the inverse function of $f_{m t}\left(\underline{Y}_{m t}^{S^{q}}\right)$. Hence $c_{m t}^{S^{q}}$ is uniquely decided, $f_{m t}^{-1}\left(Y_{m t}^{S^{q}}\right)$, such that

$$
c_{m t}^{S^{q}}=f_{m t}^{-1}\left(\frac{f_{m t}\left(\bar{Y}_{m t}^{S^{q}}\right)-f_{m t}\left(\underline{Y}_{m t}^{S^{q}}\right)}{\bar{Y}_{m t}^{S^{q}}-\underline{Y}_{m t}^{S^{q}}}\right),
$$

and we define

$$
\begin{aligned}
G_{m t}^{S^{q}}\left(Y_{m t}^{S^{q}}\right):= & \frac{f_{m t}\left(\bar{Y}_{m t}^{S^{q}}\right)-f_{m t}\left(\underline{Y}_{m t}^{S^{q}}\right)}{\bar{Y}_{m t}^{S^{q}}-\underline{Y}_{m t}^{S^{q}}}\left(Y_{m t}^{S^{q}}-c_{m t}^{S^{q}}\right) \\
& +f_{m t}\left(c_{m t}^{S^{q}}\right), \\
L_{m t}^{S^{q}}\left(Y_{m t}^{S^{q}}\right):= & \begin{cases}G_{m t}^{S^{q}}\left(Y_{m t}^{S^{q}}\right) & \left(f_{m t}^{\prime \prime}(y)>0\right) \\
F_{m t}^{S^{q}}\left(Y_{m t}^{S^{q}}\right) & \left(f_{m t}^{\prime \prime}(y)<0\right) .\end{cases}
\end{aligned}
$$

By the definition, $f_{m t}\left(Y_{m t}^{S^{q}}\right) \geq L_{m t}\left(Y_{m t}^{S^{q}}\right)$.

For all $y \in S^{q}, \mu_{m}(y):=\sum_{t=1}^{T_{m}} f_{m t}(y) \geq \sum_{t=1}^{T_{m}} L_{m t}^{S^{q}}(y)$.

Let $L_{m}^{S^{q}}(y):=\sum_{t=1}^{T_{m}} L_{m t}^{S^{q}}(y)\left(0 \leq m \leq M+2\left(P+\sum_{k=1}^{M} P_{k}\right)\right)$. Then $L_{0}(y)$ is a linear function which is lower function for the convex envelope of $\mu_{0}(y)$ on the rectangle.

$\left(\operatorname{LRP}\left(S^{q}\right)\right)$ is the linear problem of $(P 2)$ by the lower bounded function of $\mu_{m}(y)$ :

$$
\begin{array}{ll}
\min & L_{0}^{S^{q}}(y) \\
\text { s.t. } & L_{m}^{S^{q}}(y) \leq 0 \\
& \left(m=1,2, \ldots, M+2\left(P+\sum_{k=1}^{M} P_{k}\right)\right) .
\end{array}
$$

By the definition $\left(\operatorname{LRP}\left(S^{q}\right)\right)$, any $y$ in $S^{q}$ satisfying the restricted condition of $(P 2)$ satisfy the restricted condition of $\left(\operatorname{LRP}\left(S^{q}\right)\right)$.

Lemma 2. The value of $\left(\operatorname{LRP}\left(S^{q}\right)\right)$ is less than the optimal value for the problem $(P 2)$ on $S^{q}$.
Proof. The definition of $\left(\operatorname{LRP}\left(S^{q}\right)\right)$ implies the statement naturally.

Lemma 3. Assume that $S^{q+1} \subset S^{q} \subset \cdots \subset S^{0} \subset R^{N+P_{\text {sum }}}$, and $\bigcap_{q=0}^{\infty} S^{q}=\left\{y^{*}\right\}$. For each $m=0,1,2, \ldots, M+2\left(P+\sum_{k=1}^{M} P_{k}\right)$ and $t=1,2, \ldots, T_{m}, \lim _{q \rightarrow \infty} \max _{y \in S^{q}}\left|F_{m t}^{S^{q}}(y)-f_{m t}(y)\right|=0$ and $\lim _{q \rightarrow \infty} \max _{y \in S^{q}}\left|G_{m t}^{S^{q}}(y)-f_{m t}(y)\right|=0$.

Proof. Let $y^{q}:=\left\{y \in S^{q} \mid \min \left(\lambda_{m t i} y_{i}^{q}, \lambda_{m t i} \bar{y}_{i}^{q}\right)(i=1, \ldots\right.$, $\left.\left.N+P_{\text {sum }}\right)\right\}$ and $\bar{y}^{q}:=\left\{y \in S^{q} \mid \max \left(\lambda_{m t i} y_{i}^{q}, \lambda_{m t i} \bar{y}_{i}^{q}\right)(i=\right.$ $\left.\left.1, \ldots, N+P_{\text {sum }}\right)\right\}$.

Since $\bigcap_{q=0}^{\infty} S^{q}=\left\{y^{*}\right\}$, the values $\underline{y}^{q}$ and $\bar{y}^{q}$ satisfy $\lim _{q \rightarrow \infty} \underline{y}^{q}=\lim _{q \rightarrow \infty} \bar{y}^{q}=y^{*}$.

Hence, $\bar{Y}_{m t}^{S^{q}}-\underline{Y}_{m t}^{S^{q}}=\sum_{i=1}^{N+P_{\text {sum }}}\left|\lambda_{m t i}\right|\left(\bar{y}_{i}^{q}-\underline{y}_{i}^{q}\right) \stackrel{q \rightarrow \infty}{\longrightarrow} 0$.

Now,

$$
\begin{aligned}
& \left|F_{m t}^{S^{q}}(y)-f_{m t}(y)\right| \\
& =\left|F_{m t}^{S^{q}}\left(Y_{m t}^{S^{q}}\right)-f_{m t}\left(Y_{m t}^{S^{q}}\right)\right| \\
& =\mid \frac{f_{m t}\left(\bar{Y}_{m t}^{S^{q}}\right)-f_{m t}\left(\underline{Y}_{m t}^{S^{q}}\right)}{\bar{Y}_{m t}^{S^{q}}-\underline{Y}_{m t}^{S^{q}}}\left(Y_{m t}^{S^{q}}-\underline{Y}_{m t}^{S^{q}}\right) \\
& +f_{m t}\left(\underline{Y}_{m t}^{S^{q}}\right)-f_{m t}\left(Y_{m t}^{S^{q}}\right) \mid .
\end{aligned}
$$

The function $\left|F_{m t}^{S^{q}}(y)-f_{m t}(y)\right|$ is concave on $\left[\underline{Y}_{m t}^{S^{q}}, \bar{Y}_{m t}^{S^{q}}\right]$; therefore $c_{m t}^{S^{q}}$ attains the maximum value of $\left|F_{m t}^{S^{q}}(y)-f_{m t}(y)\right|$ : $\max _{y \in S^{q}}\left|F_{m t}^{S^{q}}(y)-f_{m t}(y)\right|$

$$
\begin{aligned}
\max _{y \in S^{q}} & \left|F_{m t}^{S^{q}}(y)-f_{m t}(y)\right| \\
= & \left|F_{m t}^{S^{q}}\left(c_{m t}^{S^{q}}\right)-f_{m t}\left(c_{m t}^{S^{q}}\right)\right| \\
= & \mid \frac{f_{m t}\left(\bar{Y}_{m t}^{S^{q}}\right)-f_{m t}\left(\underline{Y}_{m t}^{S^{q}}\right)}{\bar{Y}_{m t}^{S^{q}}-\underline{Y}_{m t}^{S^{q}}}\left(c_{m t}^{S^{q}}-\underline{Y}_{m t}^{S^{q}}\right) \\
& +f_{m t}\left(\underline{Y}_{m t}^{S^{q}}\right)-f_{m t}\left(c_{m t}^{S^{q}}\right) \mid .
\end{aligned}
$$

We denote

$$
I_{m t}^{S^{q}}=\bar{Y}_{m t}^{S^{q}}-\underline{Y}_{m t}^{S^{q}}, \quad c_{m t}^{S^{q}}=\underline{Y}_{m t}^{S^{q}}+\theta_{m t}^{S^{q}} I_{m t}^{S^{q}} \quad\left(0<\theta_{m t}^{S^{q}}<1\right) .
$$


Since $I_{m t}^{S^{q}} \rightarrow 0$ for $q \rightarrow \infty$,

$$
\begin{aligned}
& \mid \frac{f_{m t}\left(\bar{Y}_{m t}^{S^{q}}\right)-f_{m t}\left(\underline{Y}_{m t}^{S^{q}}\right)}{\bar{Y}_{m t}^{S^{q}}-\underline{Y}_{m t}^{S^{q}}}\left(c_{m t}^{S^{q}}-\underline{Y}_{m t}^{S^{q}}\right) \\
& +f_{m t}\left(\underline{Y}_{m t}^{S^{q}}\right)-f_{m t}\left(c_{m t}^{S^{q}}\right) \\
& =\mid \frac{f_{m t}\left(\underline{Y}_{m t}^{S^{q}}+I_{m t}^{S^{q}}\right)-f_{m t}\left(\underline{Y}_{m t}^{S^{q}}\right)}{\underline{Y}_{m t}^{S^{q}}+I_{m t}^{S^{q}}-\underline{Y}_{m t}^{S^{q}}}\left(\underline{Y}_{m t}^{S^{q}}+\theta_{m t}^{S^{q}} I_{m t}^{S^{q}}-\underline{Y}_{m t}^{S^{q}}\right) \\
& +f_{m t}\left(\underline{Y}_{m t}^{S^{q}}\right)-f_{m t}\left(\underline{Y}_{m t}^{S^{q}}+\theta_{m t}^{S^{q}} I_{m t}^{S^{q}}\right) \mid \\
& =\mid \frac{f_{m t}\left(\underline{Y}_{m t}^{S^{q}}+I_{m t}^{S^{q}}\right)-f_{m t}\left(\underline{Y}_{m t}^{S^{q}}\right)}{\underline{Y}_{m t}^{S^{q}}+I_{m t}^{S^{q}}-\underline{Y}_{m t}^{S^{q}}}\left(\theta_{m t}^{S^{q}} I_{m t}^{S^{q}}\right) \\
& +f_{m t}\left(\underline{Y}_{m t}^{S^{q}}\right)-f_{m t}\left(\underline{Y}_{m t}^{S^{q}}+\theta_{m t}^{S^{q}} I_{m t}^{S^{q}}\right) \\
& =\mid f_{m t}\left(\underline{Y}_{m t}^{S^{q}}+I_{m t}^{S^{q}}\right) \theta_{m t}^{S^{q}}-f_{m t}\left(\underline{Y}_{m t}^{S^{q}}\right) \theta_{m t}^{S^{q}}+f_{m t}\left(\underline{Y}_{m t}^{S^{q}}\right) \\
& -f_{m t}\left(\underline{Y}_{m t}^{S^{q}}+\theta_{m t}^{S^{q}} I_{m t}^{S^{q}}\right) \\
& \stackrel{q \rightarrow \infty}{\longrightarrow} \mid f_{m t} \underline{Y}_{m t}^{S^{q}} \theta_{m t}^{S^{q}}-f_{m t}\left(\underline{Y}_{m t}^{S^{q}}\right) \theta_{m t}^{S^{q}} \\
& +f_{m t}\left(\underline{Y}_{m t}^{S^{q}}\right)-f_{m t}\left(\underline{Y}_{m t}^{S^{q}}\right) \mid=0 .
\end{aligned}
$$

Thus

$$
\begin{aligned}
& \left|G_{m t}^{S^{q}}(y)-f_{m t}(y)\right| \\
& =\left|G_{m t}^{S^{q}}\left(Y_{m t}^{S^{q}}\right)-f_{m t}\left(Y_{m t}^{S^{q}}\right)\right| \\
& =\mid \frac{f_{m t}\left(\bar{Y}_{m t}^{S^{q}}\right)-f_{m t}\left(\underline{Y}_{m t}^{S^{q}}\right)}{\bar{Y}_{m t}^{S^{q}}-\underline{Y}_{m t}^{S^{q}}}\left(Y_{m t}^{S^{q}}-c_{m t}^{S^{q}}\right) \\
& +f_{m t}\left(c_{m t}^{S^{q}}\right)-f_{m t}\left(Y_{m t}^{S^{q}}\right) \mid .
\end{aligned}
$$

On the other hand $\left|G_{m t}^{S^{q}}(y)-f_{m t}(y)\right|$ is a convex function by the same argument, and we obtain the following $\max _{y \in S^{q}}\left|G_{m t}^{S^{q}}(y)-f_{m t}(y)\right| \rightarrow 0$ for $q \rightarrow \infty$.

Lemma 4. Under the same assumption of Lemma 3 $\max _{y \in S^{q}}\left|L_{m}^{S^{q}}(y)-\mu_{m}(y)\right| \rightarrow 0$ for $q \rightarrow \infty$ and each $m=$ $0,1,2, \ldots, M+2\left(P+\sum_{k=1}^{M} P_{k}\right)$.
Proof. Lemma 3 and the definitions $L_{m}^{S^{q}}(y), \mu_{m}(y)$ imply Lemma 4, standardly.

\section{Branch and Bound Algorithm and Its Convergence}

In Section 2, we transformed the initial problem $(P 0)$ into the equivalent problem $(P 2)$, and we make the linear relaxation problem (LRP) of (P2) to find the approximate value of $(P 2)$ easily. Now we get it by using branch and bound algorithm.

3.1. Branch and Bound Algorithm. We solve the linear relaxation problem on initial domain $S^{0}$ to get the linear optimal value as lower bound of $(P 2)$ and upper bound of $(P 2)$. For preparing to separate the active domains, we let the active domains set be $\mathbb{Q}_{q}$ and active domain $S^{q(k)} \subset S^{0} . q$ presents the times of the cutting domains and stage number and $k$ presents the number of active domains on stage $q$. If $S^{q(k)}$ is active domain, we divide $S^{q(k)}$ into half domains $S^{q(k) \cdot 1}, S^{q(k) \cdot 2}$ and linearize $(P 2)$ on each domain and solve the linear problems. After the above calculations, we get the lower and upper bound value of $(P 2)$. After the repeat calculations, we get the convergence for the sequences of the lower and upper bound values, and we get the optimal value and solution.

3.1.1. Branching Rule. We denote that $S^{q(k)}=\left\{y \mid \underline{y}_{n}^{q(k)} \leq\right.$ $\left.y_{n}^{q(k)} \leq \bar{y}_{n}^{q(k)}, n=1, \ldots, N+P_{\text {sum }}\right\} \subseteq S^{0}$. We select the branching variable $i$ such that $i=n \max \left\{\bar{y}_{n}^{q(k)}-y^{q(k)}, n=1,2\right.$, $\left.\ldots, N+P_{\text {sum }}\right\}$, and we divide the interval $\left[y_{i}^{q(k)}, \bar{y}_{i}^{q(k)}\right]$ into half intervals: $\left[\underline{y}_{i}^{q(k)},\left(\underline{y}_{i}^{q(k)}+\bar{y}_{i}^{q(k)}\right) / 2\right]$ and $\left[\left(\underline{y}_{i}^{q(k)}+\bar{y}_{i}^{q(k)}\right) / 2, \bar{y}_{i}^{q(k)}\right]$.

\subsubsection{Algorithm Statement}

Step 0 . Firstly, we let $q$ be 0 and let $k$ be 1 . And we set an appropriate $\epsilon$-value as a convergence tolerance, the initial upper bound $V^{*}=\infty$, and $\widehat{Q}_{0}=S^{0(1)}$. We solve $\operatorname{LRP}\left(S^{0(1)}\right)$, and we denote the linear optimal solution and optimal value by $\widehat{y}\left(S^{0(1)}\right)$ and $\mathrm{LB}_{0(1)}$. If $\widehat{y}\left(S^{0(1)}\right)$ is feasible for $(P 2)$, then update $V^{*}=\mu_{0}\left(\widehat{y}\left(S^{0(1)}\right)\right)$ and we set the initial lower bound $\mathrm{LB}=$ $\mathrm{LB}_{0(1)}$. If $V^{*}-\mathrm{LB} \leq \epsilon$, then we get the $\epsilon$-approximate optimal value $\mu_{0}\left(\widehat{y}\left(S^{0(1)}\right)\right)$ and optimal solution $\widehat{y}\left(S^{0(1)}\right)$ of $(P 2)$, so we stop this algorithm. Otherwise, we proceed to Step 1.

Step 1. For all $k$, we divide $S^{q(k)}$ to get two half domains, $S^{q(k) \cdot 1}$ and $S^{q(k) \cdot 2}$, according to the above branching rule.

Step 2. For all $k$ and each domain $S^{q(k) \cdot v}(v=1,2)$, we calculate

$$
\begin{array}{r}
\underline{\mu}_{m}(v)=\sum_{t=1, c_{m t}>0}^{\Gamma_{m}} c_{m t} \exp \left(\underline{Y}_{m t}^{S^{q(k) \cdot v}}\right)+\sum_{t=1, c_{m t}<0}^{\Gamma_{m}} c_{m t} \exp \left(\bar{Y}_{m t}^{S^{q(k) \cdot v}}\right) \\
\left(m=1, \ldots, M+2\left(P+\sum_{k=1}^{M} P_{k}\right)\right),
\end{array}
$$

where $c_{m t}, \underline{Y}_{m t}^{S^{q(k) \cdot v}}$, and $\bar{Y}_{m t}^{S^{q(k) \cdot v}}$ are defined in Section 2.3. 
If there is the $\underline{\mu}_{m}(v)$ that satisfy $\underline{\mu}_{m}(v)>0$ for some $m \in$ $\left\{1,2, \ldots, M+2\left(P+\sum_{k=1}^{M} P_{k}\right)\right\}, S^{q(k) v}$ is infeasible domain for $(P 2)$, then we delete the domain from $Q_{q}$. If $S^{q(k) \cdot v}(v=1,2)$ are all deleted for all $k$, then the problem has no feasible solutions.

Step 3. For left domains, we compute $A_{m t}^{S^{q(k) \cdot v}}, B_{m t}^{S^{(q(k) \cdot v}}, \underline{Y}_{m t}^{S^{q(k) \cdot v}}$, and $\bar{Y}_{m t}^{S^{q(k) \cdot v}}$ as defined in Sections 2.2 and 2.3. We solve the $\operatorname{LRP}\left(S^{q(k) \cdot v}\right)$ by simplex algorithm, and we denote the obtained linear optimal solutions and values by $\left(\hat{y}\left(S^{q(k) \cdot v}\right)\right.$, $\left.\mathrm{LB}_{q(k) \cdot v}\right)$. Then if $\hat{y}\left(S^{q(k) \cdot v}\right)$ is feasible for $(P 2)$, we update $V^{*}=\min \left\{V^{*}, \mu_{0}\left(\widehat{y}\left(S^{q(k) \cdot v}\right)\right)\right\}$. If $\mathrm{LB}_{q(k) \cdot v}>V^{*}$, then delete the corresponding domains from $\mathbb{Q}_{q}$. If $V^{*}-\mathrm{LB}_{q(k) \cdot v} \leq \epsilon$, then we get the $\epsilon$-approximate optimal value $\mu_{0}\left(\hat{y}\left(S^{q(k) \cdot v}\right)\right)$ and optimal solution $\hat{y}\left(S^{q(k) \cdot v}\right)$ of $(P 2)$, so we stop this algorithm. Otherwise, we proceed to Step 4.

Step 4. We update the index of left domains $S^{q(k) \cdot v}$ to $S^{q+1(k)}$; then we initialize $k$. And we settle that $\mathbb{Q}_{q+1}$ is a set of $S^{q+1(k)}$, and go to Step 1.

3.2. The Convergence of the Algorithm. Corresponding to [4], we obtain the convergence of the algorithm (cf. [4]).

Theorem 5. Suppose that problem (P2) has a global optimal solution, and let $\mu_{0}^{*}$ be the global optimal value of (P2). Then one has the following:

(i) for the case $\epsilon>0$, the algorithm always terminates after finitely many iterations yielding a global $\epsilon$-optimal solution $y^{*}$ and a global $\epsilon$-optimal value $V^{*}$ for problem $(P 2)$ in the sense that

$y^{*} \in S, \quad V^{*}-\epsilon \leq \mu_{0}^{*}$ with $V^{*}=\mu_{0}\left(y^{*}\right) ;$

(ii) for the case $\epsilon \rightarrow 0$, we assume the sequence $\epsilon_{n}$ is convergence tolerance, such that $\epsilon_{1}>\epsilon_{2}>, \ldots,>\epsilon_{n}>$ $\epsilon_{n+1}>, \ldots,>0$; that is, $\lim _{n \rightarrow \infty} \epsilon_{n}=0$. And we assume the sequence $y_{n}^{*}$ is optimal solution of $(P 2)$ corresponding to $\epsilon_{n}$. Then the accumulation point of $y_{n}^{*}$ is global optimal solution of (P2).

Proof. (i) It is obvious by algorithm statement. is $V_{n}^{*}$ :

(ii) We assume that the upper bound corresponding to $\epsilon_{n}$

$$
\mu_{0}\left(y_{n}^{*}\right) \in\left[V_{n}^{*}-\epsilon, V_{n}^{*}\right] ;
$$

$y_{n}^{*}$ is the point sequence on bounded closed set, so $y_{n}^{*}$ has a converge subsequence $y_{n i}^{*}$. We assume that $\lim _{i \rightarrow \infty} y_{n i}^{*}=y^{*}$; then

$$
\begin{aligned}
& V_{n i}^{*}-\epsilon_{n i} \leq \mu_{0}\left(V_{n i}^{*}\right) \leq V_{n i}^{*} \quad i \longrightarrow \infty \\
& \text { then } n i \longrightarrow \infty, \text { so } \lim _{n i \rightarrow \infty} \epsilon_{n i}=0 .
\end{aligned}
$$

$V_{n}^{*}$ is monotone decreasing sequence, so it converges. We assume that $\lim _{n \rightarrow \infty} V_{n}^{*}=\mu_{0}^{*}$ :

$$
\lim _{i \rightarrow \infty}\left(V_{n i}^{*}-\epsilon_{n i}\right) \leq \lim _{i \rightarrow \infty} \mu_{0}\left(y_{n i}^{*}\right) \leq \lim _{i \rightarrow \infty} V_{n i}^{*} ;
$$

$\mu_{0}(y)$ is continuous function, so $\lim _{i \rightarrow \infty} \mu_{0}\left(y_{n i}\right)=\mu_{0}\left(y^{*}\right)$. And $\mu_{0}^{*} \leq \mu_{0}^{y^{*}} \leq \mu_{0}^{*}$; that is, $\mu_{0}\left(y^{*}\right)=\mu_{0}^{*}$. For $\forall m, \mu_{m}\left(y_{n}^{*}\right) \leq 0$. As $\mu_{m}\left(y^{*}\right)$ is continuous, $\lim _{n \rightarrow \infty} \mu_{m}\left(y_{n}^{*}\right)=\mu_{m}\left(y^{*}\right) \leq 0$.

\section{Numerical Experiment}

In this chapter, we show the numerical experiments of these optimization problems according to the former rules. We make the algorithms coded with MATLAB. In these codes, we use MATLAB's unique function code "linprog" to solve the linear optimization problems.

Example 1. Consider

$$
\begin{array}{cc}
\min \quad h(x)= & \sin \left(\frac{x_{1}^{2}+3 x_{2}-2 x_{2}^{2}+1}{x_{1}^{2}+x_{2}+2}\right) \\
& +\cos \left(\frac{-x_{2}^{2}+2 x_{1}+2 x_{2}}{x_{1}+2.5}\right) \\
\text { s.t. } \quad & x_{1}^{2}-\frac{x_{1}}{x_{2}}-1 \leq 0 \\
& x_{1}+3 \frac{x_{2}}{x_{1}}-5 \leq 0 \\
& X=\left\{x: 1 \leq x_{1} \leq 3,1 \leq x_{2} \leq 3\right\} .
\end{array}
$$

We set $\epsilon=0.0001$. After the algorithm, we found a global $\epsilon$-optimal value $V^{*}=1.0748$ when the global $\epsilon$-optimal solution is $\left(x_{1}, x_{2}\right)^{T}=(1.34977,1.64232)$.

Example 2. Consider

$$
\begin{array}{ll}
\min & \exp \left(\frac{-x_{1}^{2}+3 x_{1}+2 x_{2}^{2}+3 x_{2}+3.5}{x_{1}+1}\right) \\
& -\exp \left(\frac{x_{2}}{x_{1}^{2}-2 x_{1}+x_{2}^{2}-8 x_{2}+20}\right) \\
\text { s.t. } & x_{1}-\frac{x_{2}}{x_{1}} \leq 1 \\
& 2 \frac{x_{1}}{x_{2}}+x_{2}+\leq 6 \\
& 2 x_{1}+x_{2} \leq 8 \\
& X=\left\{x: 1 \leq x_{1} \leq 3,1 \leq x_{2} \leq 3\right\} .
\end{array}
$$

We set $\epsilon=0.0001$. After the algorithm, we found a global $\epsilon$-optimal value $V^{*}=58.2723$ when the global $\epsilon$-optimal solution is $\left(x_{1}, x_{2}\right)^{T}=(1,1.6180)$.

Example 3. Consider

$$
\begin{aligned}
\min & \sin \left(\frac{x_{1}^{2}+2 x_{2}-2 x_{1}+x_{2}^{2}+1}{x_{1}+x_{2}^{2}+2}\right) \\
& +\cos \left(\frac{3 x_{1}^{2}-3 x_{2}+2 x_{1}+x_{2}^{2}+5}{x_{1}^{2}+2 x_{2}^{2}+10}\right)
\end{aligned}
$$




$$
\begin{array}{ll}
\text { s.t. } & \sin \left(\frac{x_{1}^{2}+3 x_{2}-2 x_{2}^{2}+2}{x_{1}^{2}+x_{2}+2}\right) \\
& +\cos \left(\frac{-x_{2}^{2}+2 x_{1}+2 x_{2}}{x_{1}+2.5}\right) \leq 2 \\
& X=\left\{x: 1 \leq x_{1} \leq 2,1 \leq x_{2} \leq 2\right\} .
\end{array}
$$

We set $\epsilon=0.0001$. After the algorithm, we found a global $\epsilon$-optimal value $V^{*}=1.09133$ when the global $\epsilon$-optimal solution is $\left(x_{1}, x_{2}\right)^{T}=(2,1)$.

Example 4. Consider

$$
\begin{array}{ll}
\min \quad \exp \left(\frac{x_{1}^{2}-2 x_{2}^{2}+8}{2 x_{1}^{2}+x_{2}+1}\right) \\
\quad+\exp \left(\frac{3 x_{1}-x_{2}^{2}+5}{x_{1}^{2}-x_{1}+x_{2}^{2}-3 x_{2}+10}\right) \\
\text { s.t. } \quad x_{1}^{2}-2 x_{2} \leq 1 \\
\quad x_{1}-\frac{x_{2}}{x_{1}} \leq 1 \\
\quad 2 x_{1}+x_{2}^{2} \leq 6 \\
\quad X=\left\{x: 1.5 \leq x_{1} \leq 2,1.5 \leq x_{2} \leq 2\right\} .
\end{array}
$$

We set $\epsilon=0.0001$. After the algorithm, we found a global $\epsilon$-optimal value $V^{*}=3.9378$ when the global $\epsilon$-optimal solution is $\left(x_{1}, x_{2}\right)^{T}=(1.5,1.7321)$.

\section{Concluding Remarks}

In this paper, we proved that we can solve the nonconvex optimization problems which [4] cannot solve that the sum of the positive (or negative) first and second derivatives function with the variable defined by sum of polynomial fractional function by using branch and bound algorithm.

\section{Conflict of Interests}

The authors declare that there is no conflict of interests regarding the publication of this paper.

\section{Acknowledgments}

The authors would like to thank S. Pei-Ping, H. Yaguchi, and $\mathrm{S}$. Tsuyumine for their good suggestion and encouragement.

\section{References}

[1] D. Cai and T. G. Nitta, "Limit of the solutions for the finite horizon problems as the optimal solution to the infinite horizon optimization problems," Journal of Difference Equations and Applications, vol. 17, no. 3, pp. 359-373, 2011.

[2] D. Cai and T. G. Nitta, "Optimal solutions to the infinite horizon problems: constructing the optimum as the limit of the solutions for the finite horizon problems," Nonlinear Analysis: Theory, Methods \& Applications, vol. 71, no. 12, pp. e2103-e2108, 2009.

[3] R. Okumura, D. Cai, and T. G. Nitta, "Transversality conditions for infinite horizon optimality: higher order differential problems," Nonlinear Analysis: Theory, Methods \& Applications, vol. 71, no. 12, pp. e1980-e1984, 2009.

[4] S. Pei-Ping and Y. Gui-Xia, "Global optimization for the sum of generalized polynomial fractional functions," Mathematical Methods of Operations Research, vol. 65, no. 3, pp. 445-459, 2007.

[5] H. Jiao, Z. Wang, and Y. Chen, "Global optimization algorithm for sum of generalized polynomial ratios problem," Applied Mathematical Modelling, vol. 37, no. 1-2, pp. 187-197, 2013.

[6] Y. J. Wang and K. C. Zhang, "Global optimization of nonlinear sum of ratios problem," Applied Mathematics and Computation, vol. 158, no. 2, pp. 319-330, 2004. 


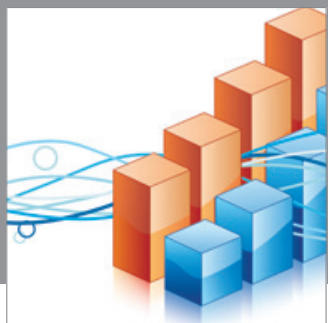

Advances in

Operations Research

mansans

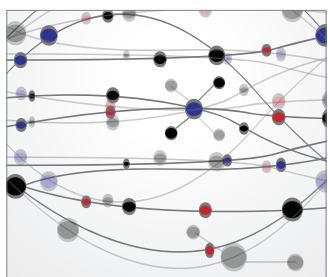

The Scientific World Journal
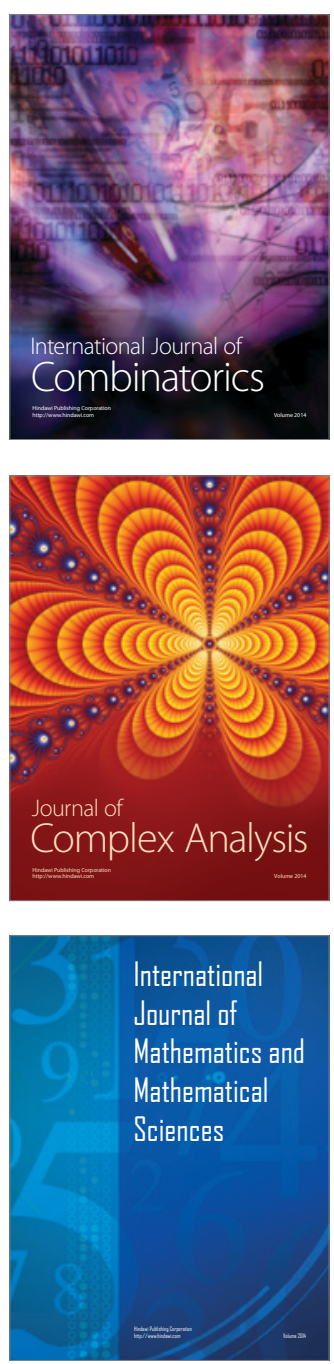
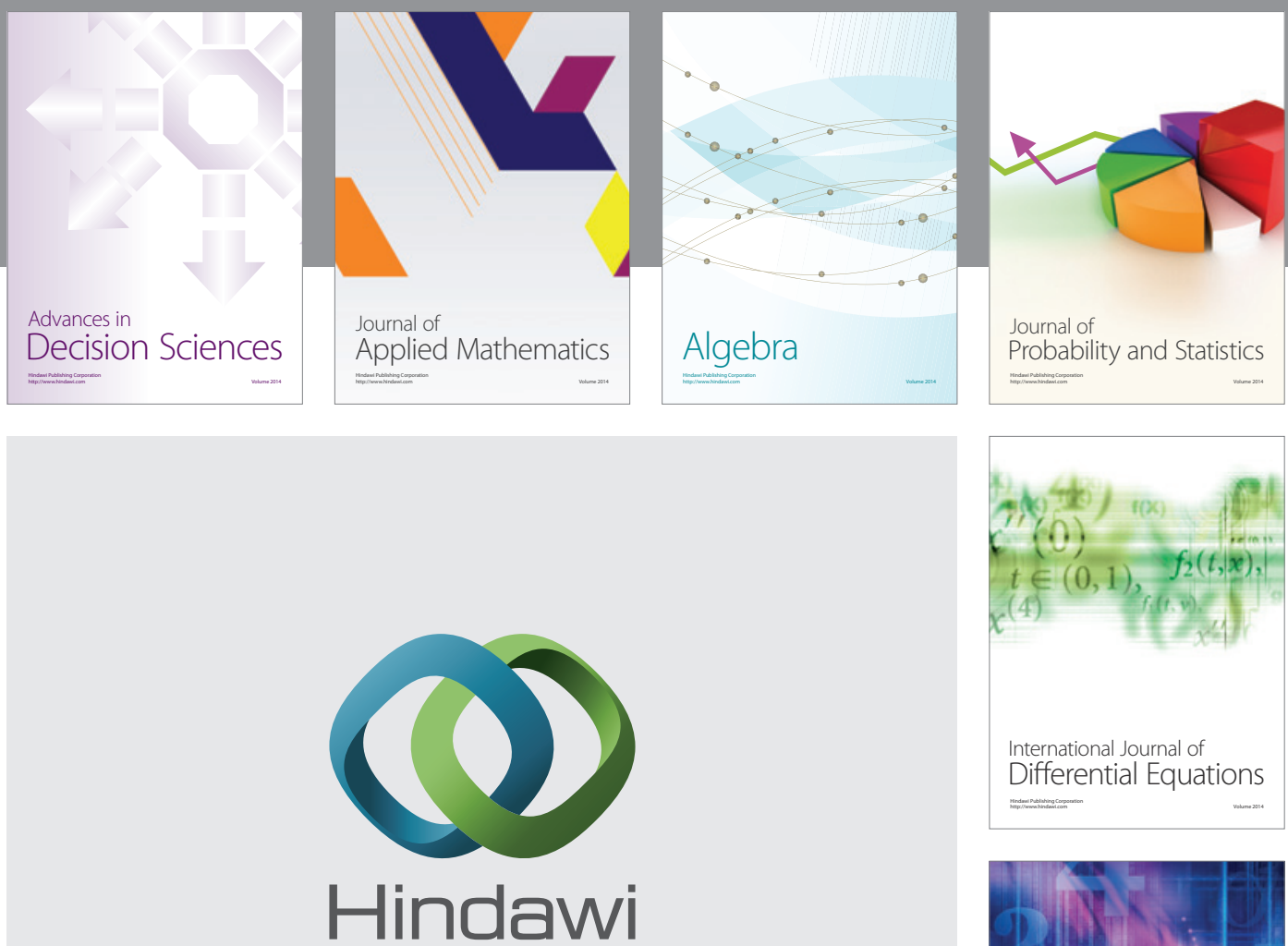

Submit your manuscripts at http://www.hindawi.com
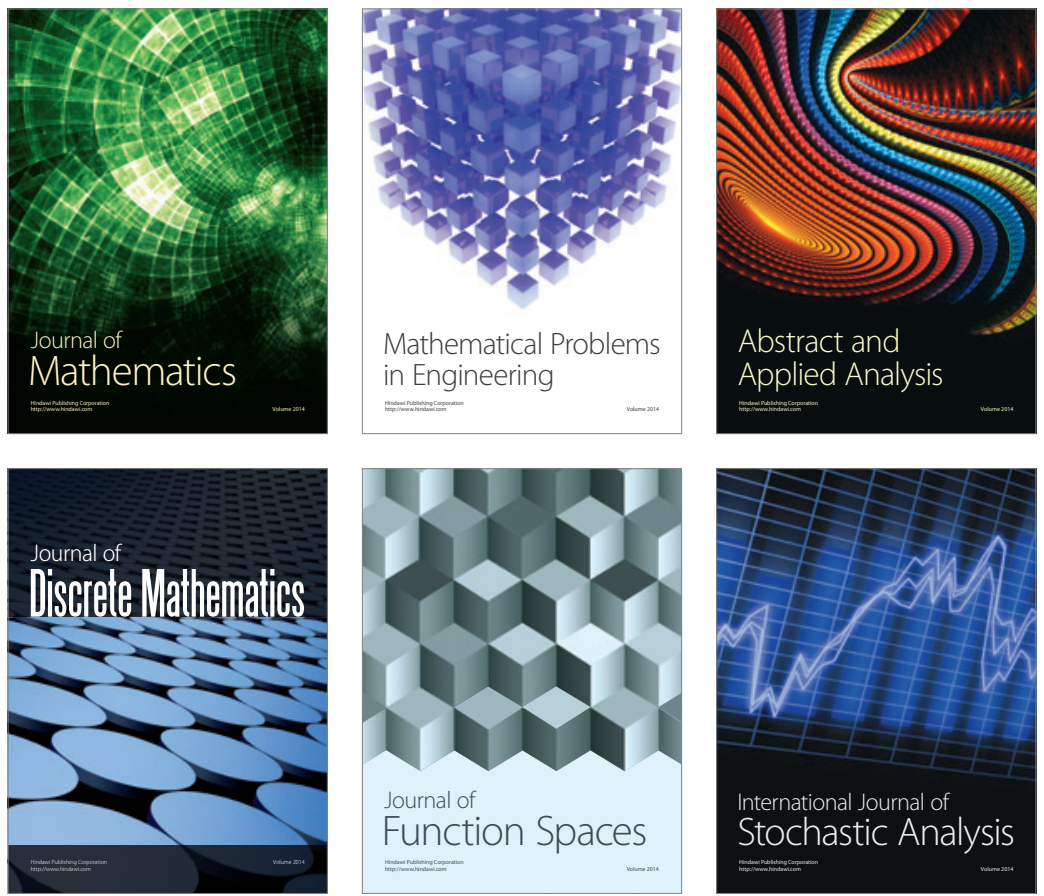

Journal of

Function Spaces

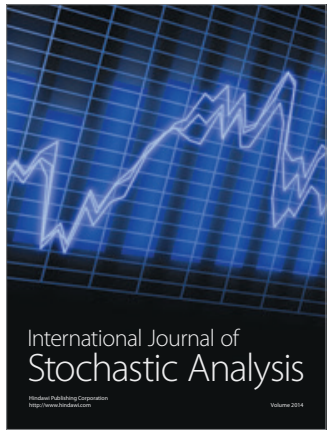

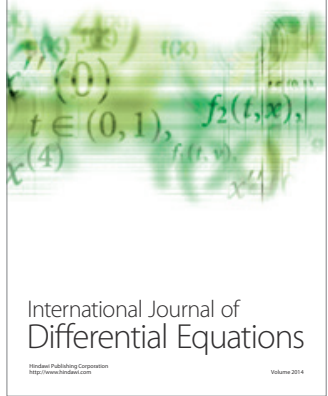
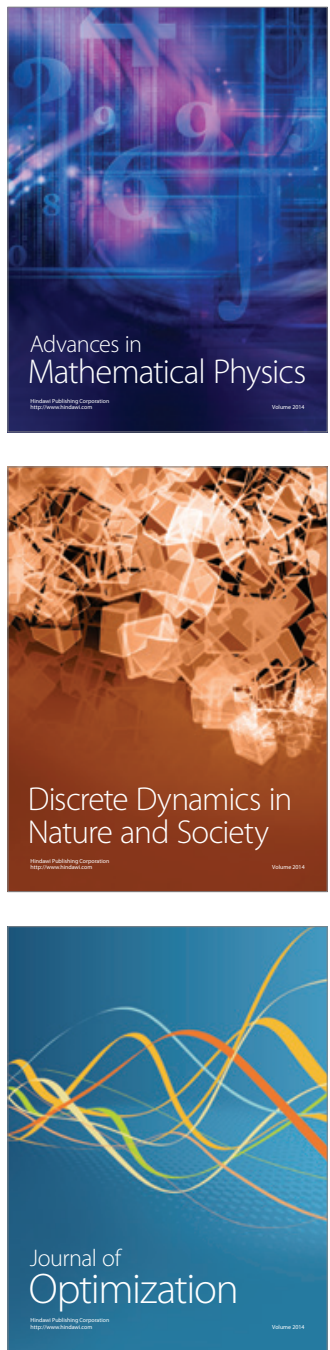